\title{
GNSS Interference in L-Band SAR Missions - Assessment and Mitigation
}

\author{
Oliver Montenbruck, Markus Markgraf \\ German Aerospace Center (DLR), German Space Operations Center (GSOC), Oberpfaffenhofen, Germany \\ Michel Tossaint \\ ESA/ESTEC, Noordwijk, The Netherlands
}

\section{BIOGRAPHY}

Dr. Oliver Montenbruck is head of the GNSS Technology and Navigation Group at DLR's German Space Operations Center (GSOC), Oberpfaffenhofen. His current research activities comprise spaceborne GNSS receiver technology, autonomous navigation systems, spacecraft formation flying and precise orbit determination as well as new constellations and multi-GNSS processing. Oliver Montenbruck presently chairs the GNSS Working Group of the International GPS Service and coordinates the performance of the MGEX Multi-GNSS Experiment. He authored numerous technical papers and various textbooks.

Markus Markgraf is a senior research engineer in the GNSS Technology and Navigation Group at DLR/GSOC. He started working at DLR in 2000 after graduating as Dipl.-Ing. (FH) for Electrical Engineering and Communication Technology. His current research activities comprise GNSS receiver technology for satellites and sounding rockets, scientific applications of GNSS and mission support and analysis. He was the key engineer for the Navigation and Occultation Experiment (NOX) on TET-1 during the design and implementation phase.

Michel Tossaint is System Engineer at the Earth Observation - Future Mission Division at ESTEC for the European Space Agency (ESA), the Netherlands. He graduated M.Sc. in Aerospace Engineering from Delft University of Technology in 1997. He joined the Space Systems Department of the Dutch NLR in 1998. In 2001 he joined ESA working on navigation related topics like EGNOS design and validation, Galileo experimentation with GIOVE satellites and GNSS evolutions in general. From 2008 he was Navigation Technical Officer in the Galileo project and evolution program working on Intersatellite Links and Galileo Second Generation design.

\section{ABSTRACT}

Synthetic Aperture Radar (SAR) satellites commonly make use of onboard Global Positioning System (GPS) receivers for precise orbit and baseline determination. In view of the extreme SAR transmit power levels, interference from
SAR signals may inhibit proper GPS tracking and poses a particular challenge to space missions using L-band SAR signals with frequencies adjacent to or even overlapping GPS frequency bands. Within this study, the impact of simulated SAR signals on direct and semi-codeless GPS signal tracking is assessed in a signal simulator test bed using two commercial-off-the-shelf geodetic-grade receivers. A high robustness of GPS tracking to both adjacent-band and in-band SAR interference is obtained within the tests using representative chirp signals. For SAR signals next to or overlapping the GPS L2 band, proper tracking of the GPS L1 C/A code, GPS2 L2C, and semi-codeless L1/L2 $\mathrm{P}(\mathrm{Y})$-code tracking is retained for interference powers up to $90 \mathrm{db}$ above the natural GPS signal power. Apparently, a high level of immunity to high-power pulsed signals with repeat periods in the (sub-)ms regime is already provided by the automatic gain control of the receivers and/or a saturation of the analog-to-digital converters in the frontend that mimic an explicit pulse blanking. On the other hand, the addition of an external pulse blanking synchronized with the chirp pulses was found to be of marginal value. This unexpected results can presumably be understood by low power "noise" in the synthetic SAR signals that adds an additional signal outside the spectral and temporal limitations of the actual chirp signal and dominates the overall interference when simulating very high chirp signal powers.

\section{INTRODUCTION}

Synthetic Aperture Radar (SAR) is a well-established technique for remote sensing of the Earth from space. SAR image generation and even more SAR interferometry requires highly precise orbit information, which is typically derived from GNSS measurements collected onboard those spacecraft. In the past, orbit determination accuracies at the few centimeter level have been demonstrated in SAR missions such as TerraSAR-X and Sentinel-1, while mmlevel accuracies have been obtained in carrier-phase based differential navigation for the TanDEM-X formation flying mission.

Dual-frequency observations, low tracking errors and 
continued availability of GNSS measurements before during and after a SAR data take are a prerequisite for achieving such performance. This can generally be achieved with properly designed spacecraft, receivers and antennas, but constitutes a substantial challenge, if the SAR instrument itself makes use of L-band signals. Among others, Lband SAR signals are of particular interest for vegetationrelated research and soil moisture mapping. In view of their wavelength, they offer a different penetration depth and thus enable access to different surfaces than $\mathrm{C}$ - and $\mathrm{X}$-band radar mission. Examples of L-band SAR missions include the Japanese ALOS/ALOS-2 satellites [1, 2] operating the Phased Array type L-band Synthetic Aperture Radar (PALSAR/PALSAR-2), as well as the upcoming Argentinian SAOCOM mission [3, 4] and the US-Indian NISAR mission [5]. In the context of SAR interferometry, SAOCOM-CS [6, 7] and TanDEM-L [8] constitute two proposed/planned dual-spacecraft formations making use of L-band SAR signals.

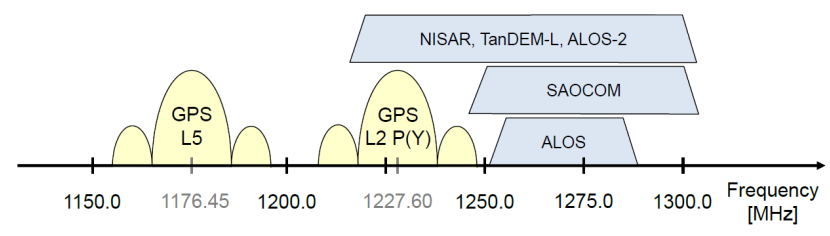

Fig. 1 Spectral range of various L-band SAR missions (blue) in relation to the GPS L2 and L5 signals (yellow). SAR spectra are shifted for clarity and amplitudes are not to scale.

In all of these missions, GNSS signal reception is at risk due to possible interference with the SAR transmission at frequencies that are adjacent to - or even overlapping - the GNSS signals in the lower L-band (Fig. 1.) Given the typical SAR transmit powers of several kilowatts $(+60 \mathrm{dBm}$ and up), even a tiny fraction thereof will result in interference power levels far above the nominal received GPS signal power of about $-130 \mathrm{dBm}$ when entering a GPS antenna or receiver. Accordingly, adequate countermeasures must be taken to ensure the availability of measurements during SAR operation. Other than narrow bandpass filter, which can only be applied for out-of-band interference and may have possible adverse impact on group and phase delays, pulse blanking has been proposed by various researchers as an effective interference mitigation technique. By way of example, the use pulse blanking has been demonstrated to counteract interference from ground-based distance measuring equipment (DME) and tactical air navigation (TACAN) transmitters in GPS L5 aviation receivers $[9,10]$, as well as interference on mass market receivers from "privacy devices" using swept signals [11].

In the context of SAR missions, pulse blanking appears particularly helpful to prevent interference and saturation of amplifiers during radar pulses generated on the host spacecraft. Here, it's use is also compatible with existing spaceborne receivers, since the timing of radar pulses is always well known and doesn't have to be inferred from the received GNSS signal. As such, the radar electronics can directly trigger an radio-frequency $(\mathrm{R} / \mathrm{F})$ switch in the antenna line and no need for hardware modification of the actual GNSS receiver arises. A first application of GPS pulse blanking in L-band SAR missions has been reported for the ALOS satellite in [1], even though the PALSAR signal is fairly well separated from the L2 center frequency. On the other hand, pulse blanking becomes more difficult, if a passive (receive-only) satellite in a formation is illuminated by the radar pulse of an active transmitter Here, the arrival time of radar pulses is not generally known and major changes of would be required to enable autonomous interference detection in existing spaceborne GNSS receivers. Complementary to the assessment of interference mitigation concepts, the characterization of the impact of SAR signals on the GPS tracking is therefore required.

Following a general presentation of SAR signals and the specific SAR signals foreseen in the aforementioned missions, the interference test setup used in our study is described. Subsequently GNSS signal tracking results for pulse blanking (with and without interference) as well as in- and out-of-band interference without blanking are discussed.

\section{SAR SIGNAL CHARACTERISTICS}

The radar concept builds on measuring the turn-around signal travel time of pulse-shaped signals reflected from a target. When using monochromatic signals, short pulses are needed to achieve a high range resolution. However, this reduces the overall duty cycle and limits the available power of the transmitted signal. To cope with this problem, modern SAR systems use "chirp" signals, which offers a higher bandwidth, and thus range resolution, independent of the pulse duration [12]. A single pulse of a typical SAR signal with center frequency $f_{0}$, pulse duration $T_{\mathrm{p}}$, and sweep rate $k$ can be represented as

$$
s(t)=\operatorname{rect}\left(\frac{t}{T}\right) \cdot \cos \left(2 \pi f_{0}+\pi k t^{2}\right) .
$$

Over the pulse duration, the instantaneous frequency

$$
f(t)=\frac{1}{2 \pi} \frac{d}{d t}\left(2 \pi f_{0}+\pi k t^{2}\right)=f_{0}+k \cdot t
$$

varies linearly between a minimum value of $f_{0}-k T / 2$ at and a maximum value of $f_{0}+k T_{\mathrm{p}} / 2$. The product $B=k T_{\mathrm{p}}$ represents the overall signal bandwidth of the SAR system and ultimately determines the range resolution. In actual operations SAR pulses with chirp modulation are transmitted continuously with a repeat period $T_{\mathrm{r}}$ that depends on the desired swath width. 
Table 1 Pulse duration $T_{\mathrm{p}}$, pulse repetition interval $T_{\mathrm{r}}$, bandwidth $B$, and center frequency $f_{0}$ of chirp modulated SAR signals in various L-band missions.

\begin{tabular}{lccccc}
\hline Mission & $\begin{array}{c}T_{\mathrm{p}} \\
{[\mu \mathrm{s}]}\end{array}$ & $\begin{array}{c}T_{\mathrm{r}} \\
{[\mu \mathrm{s}]}\end{array}$ & $\begin{array}{c}B_{\mathrm{p}} \\
{[\mathrm{MHz}]}\end{array}$ & $\begin{array}{c}f_{0} \\
{[\mathrm{MHz}]}\end{array}$ & Notes \\
\hline ALOS & $14 \ldots 40$ & $250 \ldots 667$ & 14 & 1270.0 & {$[1,2]$} \\
ALOS-2 & & & 84 & 1257.5 & {$[2]$} \\
SAOCOM & 60 & 400 & 50 & 1275.0 & {$[4]$} \\
NISAR & & & 80 & 1260.0 & {$[5]$} \\
TanDEM-L & 20 & 200 & 80 & 1257.5 & {$[13]$} \\
\hline
\end{tabular}

Parameters of SAR signals for a subset of the aforementioned L-Band SAR missions are summarized in Table 1. The center frequencies of these signals are located some $30-50 \mathrm{MHz}$ above the GPS L2 signal frequency. which allows for an at least partial overlap with the L2 signal spectrum depending on the chirp bandwidth (Fig. 1).

At a chipping rate of $10 \mathrm{MHz}$ the spectrum of the $\mathrm{P}(\mathrm{Y})$ code modulation exhibits a main lobe at $1227 \mathrm{MHz}$ flanked by the first sidelobes near $1212 \mathrm{MHz}$ and $1242 \mathrm{MHz}$. Due to a $\pm 20 \mathrm{MHz}$ bandpass filtering within the transmitting GPS satellite, the overall signal is confined to the frequency interval [1207 MHz, $1247 \mathrm{MHz}$ ], which coincides with the second nulls of the $\mathrm{P}(\mathrm{Y})$-code spectrum. The chirp signals, on the other hand, exhibit an almost constant spectral power density between $f_{0}-B / 2$ and $f_{0}+B / 2$ and a roughly exponential roll-off outside this interval.

In case of ALOS, the SAR signal spectrum is immedi- ately adjacent to, but slightly separated from the GPS L2 signal, while the SAOCOM spectrum slightly overlaps the upper L2 sidelobe. The ALOS-2, NISAR, and TanDEM$\mathrm{L}$ signals, in contrast fully cover the $\mathrm{L} 2 \mathrm{P}(\mathrm{Y})$ mainlobe as well as the spectral range of the 1-MHz L2C signal transmitted by the Block IIR-M and IIF satellites.

For completeness, Fig. 1 also shows the spectrum of the GPS L5 signal, which is shared by the Galileo E5a signal. Even in case of the windband signals of ALOS-2, NISAR, and TanDEM-L, a minimum separation of about $24 \mathrm{MHz}$ is maintained from these signals.

\section{INTERFERENCE TEST BED}

The basic architecture of the interference test bed is shown in Fig. 2. It comprises a GPS signal simulator and a programmable interference signal simulator, as well as a coupler, an R/F switch, a low noise amplifier and a pair of test receivers.

GPS signals on the L1 and L2 frequency are generated with a Spirent GSS7700 signal simulator. The simulated signals cover the civil L1 C/A and L2C signals as well as a Pseudo-Y code signal that mimics the encrypted $\mathrm{Y}$ code and is obtained from based on multiplication of the P-code with a short W-code sequence. By default, the simulator generates a GPS signal strength compatible with the minimum signal level specified for the GPS system in the early signal ICDs (approx. $-130 \mathrm{dBm}$ for satellites at a mean distance from the observer and a $0 \mathrm{~dB}$ vertical antenna gain). The overall signal level may, however, be ad-

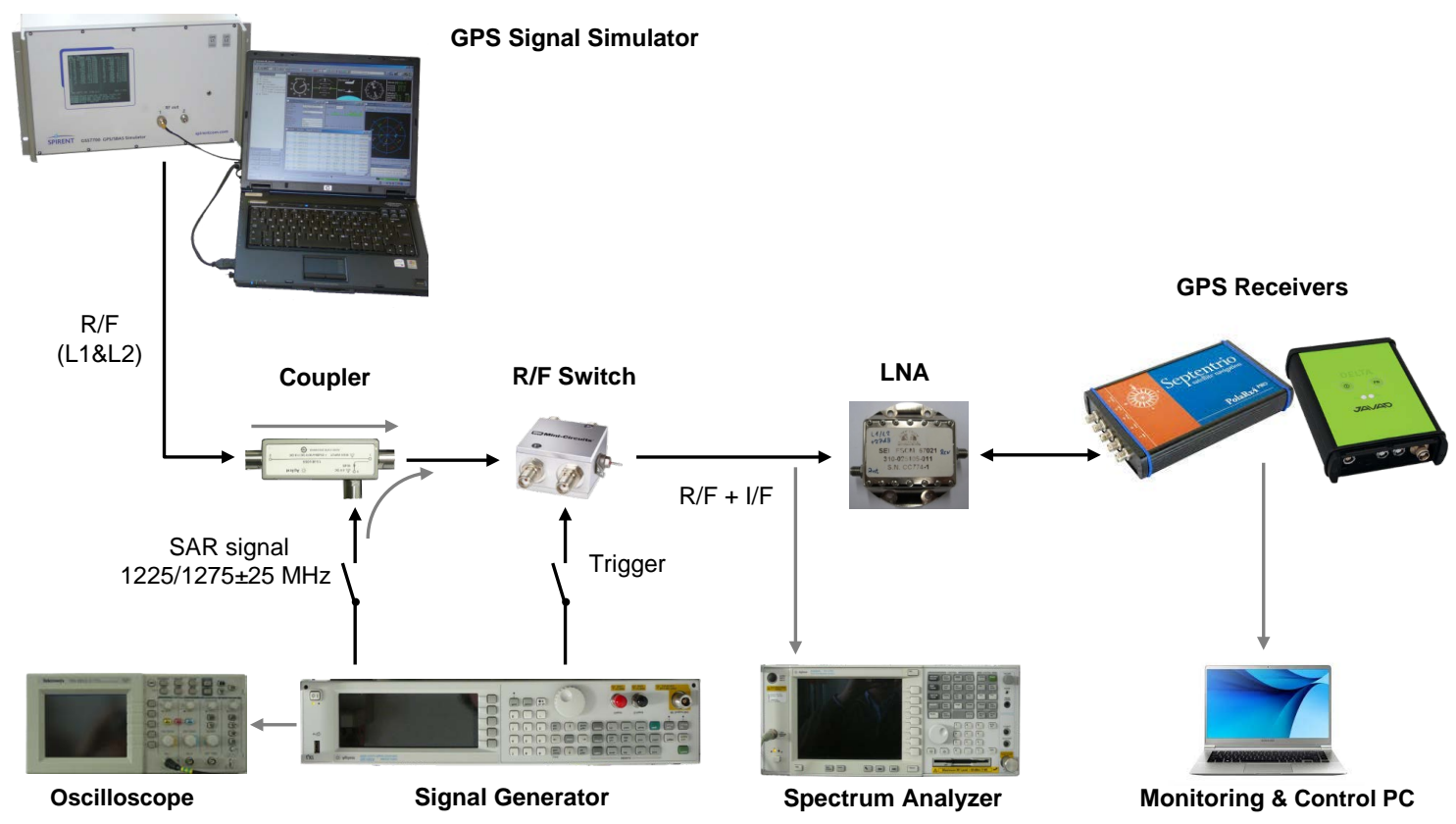

Fig. 2 Interference and pulse blanking test bed 


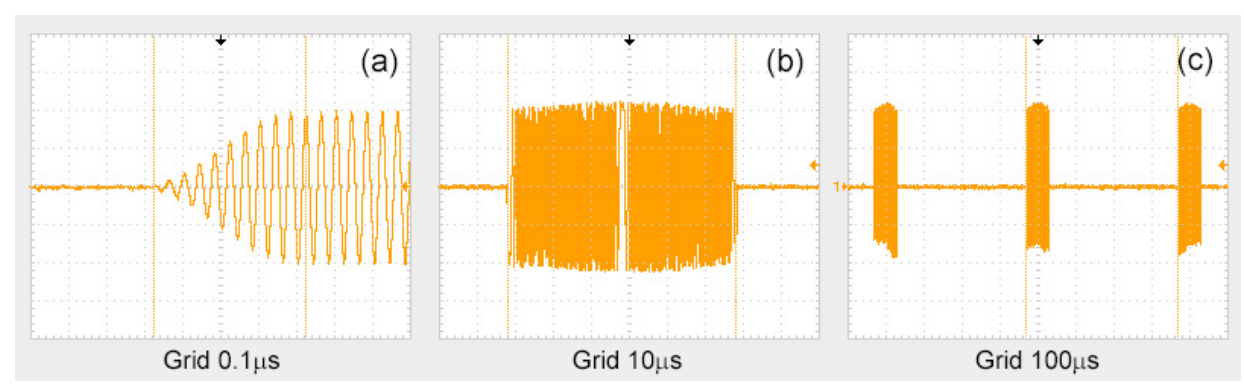

Fig. 3 Time domain view of a simulated chirp pulse train as recorded by an oscilloscope at the baseband output of the signal generator. The figures cover the $0.4 \mu \mathrm{s}$ rise time (a), the $60 \mu$ s pulse (b), and the $400 \mu$ s repeat interval (c) of a simulated SAR signal representative of the SAOCOM mission.

justed to account for the actual antenna gain (approx. 3$5 \mathrm{~dB}$ in the boresight direction) as well as the actual GPS signal strength (typically $2 \mathrm{~dB}$ higher to due conservative margins in the early link budget assumptions). In addition, a higher than normal signal level is required to compensate for the higher noise temperature experienced in simulator testing compared to the usual antenna sky temperature $[14,15]$. Finally, losses of the R/F coupler and switch need ot be taken into account. With the above considerations, a global power level increase of $15 \mathrm{~dB}$ (relative to a nominal C/A-code signal power of $-130 \mathrm{dBm}$ at the R/F outlet of the simulator) has been employed in the interference tests. It yields a peak $\mathrm{C} / \mathrm{A}$-code $\mathrm{C} / \mathrm{N}_{0}$ of about $53 \mathrm{dBHz}$ that is compatible with flight results from actual space receivers onbaord, e.g., the SWARM and Sentinel missions. The simulated GPS signal power at the LNA low noise amplifier input is later also used as reference for the simulated interference power when assessing the impact of SAR signal interference on GPS tracking.

Chirp signals with a specified center frequency, bandwidth, duration and repeat rate are generated with an Agilent N5182A MXG signal generator. Within the signal

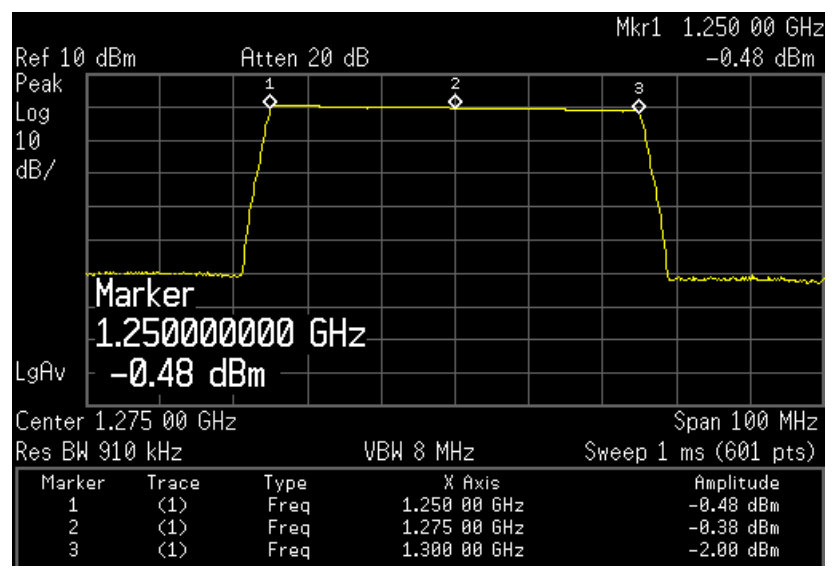

Fig. 4 Envelope spectrum of a simulated SAOCOM SAR signal. generator, the carrier is modulated with a baseband signal defined by a pre-computed set of in-phase and quadrature (I/Q) samples covering the entire pulse repeat interval $T_{\mathrm{r}}$ at a $125 \mathrm{MHz}$ sampling rate. As discussed in [16], the I/Q vector table covers the repeat period of the pulses and includes a rise and set time interval (during which the normalized signal amplitude varies between 0 and 1 according to a raised cosine profile), the actual pulse (during which the amplitude remains constant, while the frequency is varied linearly in time) and a dead time (during which the signal amplitude remains at zero). Within the SAR interference tests, rise and fall times of $0.4 \mu \mathrm{s}$ have been adopted that are sufficiently short compared to the simulated pulse durations.

For verification purposes, the in-phase component of the signal modulation has been recorded by an oscilloscope on the monitoring port of the signal generator. A sample baseband level chirp signal is illustrated in Fig. 3 based on the SAOCOM L-Band SAR characteristics. Furthermore, the corresponding envelope spectrum of the modulated signal has been obtained with a spectrum analyzer and is shown in Fig. 4. It may be recognized that the spectrum exhibits a flat top between $1250 \mathrm{MHz}$ and $1300 \mathrm{MHz}$ (i.e., $25 \mathrm{MHz}$ around the specified center frequency) and falls off at roughly $10 \mathrm{~dB} / \mathrm{MHz}$ outside this range.

The simulated SAR signal is combined with the GPS signals via a directional coupler with attenuations of $2 \mathrm{~dB}$ for the direct signal and $17 \mathrm{~dB}$ for the injected signal. An additional R/F switch prior to the low noise amplifier (LNA) can be controlled by the signal generator and allows for an optional pulse blanking synchronized to the simulated chirp pulses. The Mini-Circuits ZFSWA2-63DR+ switch used in our tests offers an isolation of better than $50 \mathrm{~dB}$ during off-times when cycled at the pulse rates and duty cycles of representative SAR signals. The blanking signal was activated slightly ahead of the actual chirp start time to account for the switching delay of typically $2 \mu$ s and to avoid a leakage of interference signals near the start of each pulse. While this form of "anticipating" switching would not be feasible in pulse-blanking based on measured inter- 
ference levels it is well compatible with the envisaged pulse blanking on the transmitting SAR satellite.

The Spectrum Microwave Model 310-025105 low noise amplifier offers a $26 \mathrm{~dB}$ amplification and uses a pair of double-diplexed ceramic filters with a bandwidth of about $\pm 40 \mathrm{MHz}$ and center frequencies of $1575 \mathrm{MHz}$ and $1227 \mathrm{MHz}$ to provide a basic level of out-of-band interference protection. Use of a standalone LNA along with a passive antenna is representative of most space missions and limits the thermal and particle radiation exposure of the electronic components. While slightly disadvantageous in terms of overall noise figure, it enables the use of filters or $\mathrm{R} / \mathrm{F}$ switches for pulse blanking prior to the LNA and therefore helps to avoid a possible saturation of the amplifier in case of high-power jamming. In accord with these considerations, the R/F switch is likewise inserted before the LNA in our test setup (Fig. 2).

In the absence of fully dedicated space receivers, two different types of geodetic-grade GNSS receivers have been used to test the resistance against SAR interference and the efficiency of external pulse blanking. These include a Septentrio PolaRx-2 GPS receiver supporting L1 C/A and L1/L2 P(Y) tracking, as well as a Javad TR-G3T multiGNSS receiver, which supports L2C tracking (as well as numerous others) in addition to the aforementioned signals.

The PolaRx2 receiver has been selected because of a high communality of its GRECO correlator chip set with the AGGA-2/4 chip used in European space GPS/GNSS receivers. Among others, an AGGA-2 based Lagrange GPSonly receiver will be flown on board the SAOCOM spacecraft. A next-generation PODRIX GPS/Galileo receiver (combining AGGA-2 and -4 chip sets) had been foreseen in the design study of a SAOCOM-CS companion satellite and is also a candidate for the TanDEM-L mission. Both the PolaRx2 receiver and the aforementioned space receivers make use of an ESA-developed technique for semicodeless tracking of the GPS P(Y)-code [17], which represents an enhanced version of Ashtechs Z-tracking [18]. Performance characteristics of the PolaRx 2 receiver ar described in [15] while actual flight results from a technology demonstration mission are reported in [19].

The Javad Delta receiver is likewise foreseen for future space missions and has been qualified for use onboard the International Space Station as part the Atomic Clock Ensemble in Space (ACES) project [20]. To enable tests under actual low Earth orbit (LEO) signal dynamics, the altitude and speed limits were removed in our test devices.

\section{ADJACENT BAND INTERFERENCE}

In a first test, we considered a case of adjacent-band interference with SAR signal frequencies next to the GPS L2 signal band. More specifically, chirp signals with the characteristics of the SAOCOM SAR signal were simulated, which cover a frequency range of $1250-1300 \mathrm{MHz}$ at a $60 \mu$ s pulse duration and a $400 \mu$ s repeat rate (see Table 1 and Fig. 1).

To study the impact of the simulated signals on the GPS tracking of the test receivers, the interference was periodically toggled on/off and varied in power after several cycles over two hours of a terrestrial scenario simulation. By way of example, Fig. 5 shows the $\mathrm{C} / \mathrm{N}_{0}$ variation of the TR-G3T receiver and the $\mathrm{C} / \mathrm{N}_{0}$ variation relative to a test without interference for a selected GPS satellite at mid elevation. Steps in $C / \mathrm{N}_{0}$ relate to the regular activation/deactivation of the interference source at time intervals of about five minutes. Following two on/off cycles at the same power level, the interference power was incremented in steps of $5 \mathrm{~dB}$ between $65 \mathrm{~dB}$ and $100 \mathrm{~dB}$ relative to the simulated power level of the GPS C/A code signals. These values refer to the peak power during the pulse on-time. Mean values of the interference signal over the pulse repeat interval are roughly seven times $(8.2 \mathrm{~dB})$ lower when considering the $15 \%$ pulse duty cycle.

The degradation of measured carrier-to-noise-density ratios with increasing jamming power is illustrated in Fig. 6 for the different signals tracked by the test devices. Even though a similar trend can be recognized for both receiver types, the comparison suggests a slightly lower interference sensitivity for the TR-G3T receiver. This difference may in part be related to different bandwidths of receiver-internal bandpass filters but may also relate to the specific $\mathrm{C} / \mathrm{N}_{0}$ estimation algorithms employed in the two receivers. Among others, such differences in the $\mathrm{C} / \mathrm{N}_{0}$ estimators are evidenced by a different scatter as well as different convergence times of the measured $\mathrm{C} / \mathrm{N}_{0}$ after step changes in the interference level.

As expected from the close proximity of the simulated SAR signal frequency, L2 tracking is clearly more sensitive to interference than L1 tracking in the given test. Nevertheless, degradations of up to $7 \mathrm{~dB}$ can also be noted in the L1 $\mathrm{C} / \mathrm{A}$ code tracking at high interference levels. These might be related to a saturation of the LNA or the automated gain control (AGC) of the employed receivers but have not been analyzed further.

With respect to $\mathrm{L} 2 \mathrm{C}$ tracking, the $\mathrm{C} / \mathrm{N}_{0}$ loss for this signal due to interference is clearly lower than that of the L2 P(Y), but still substantially larger than that of L1 C/A, which benefits from the much larger frequency separation. Comparing the $\mathrm{C} / \mathrm{N}_{0}$ loss for the three signals, it may be recognized that the loss for $\mathrm{P}(\mathrm{Y})$ tracking closely matches the sum of the L1 C/A and L2C losses (when all values are expressed in $[\mathrm{dB}])$. This relation is indeed to be expected from the theory of semi-codeless tracking [18] and empirically confirmed by the current test results. Since L1 losses are generally limited except for very high interference levels when considering SAR signals in the lower Lband, both L2C and L2 P(Y) carrier phase observations can 


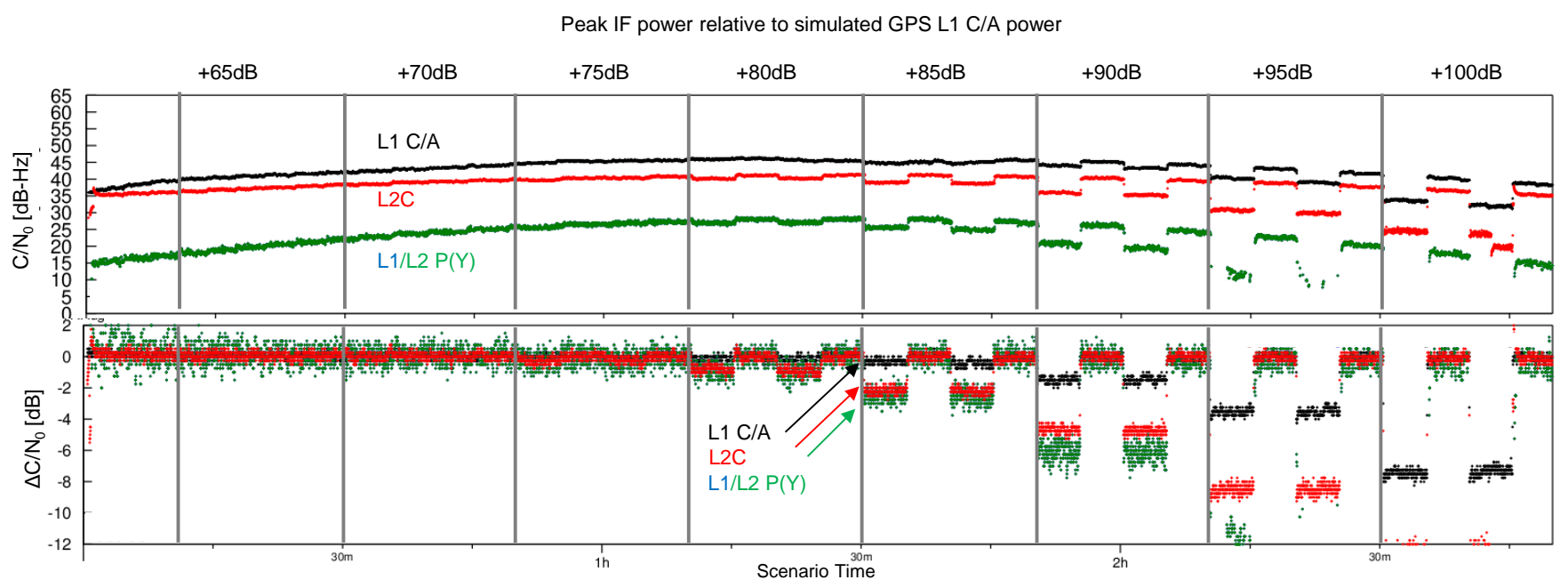

Fig. 5 Measured carrier-to-noise density variation (top) of the Javad TR-G3T receiver and difference w.r.t. to an interference-free simulation (bottom) during on-off toggling of SAOCOM-like chirp signals at different power levels relative to the simulated GPS signals.

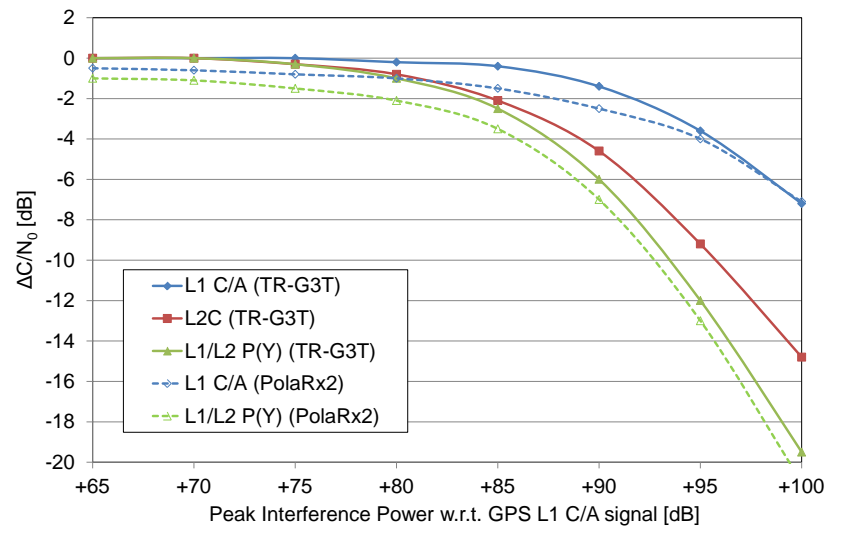

Fig. $6 \mathrm{C} / \mathrm{N}_{0}$ degradation of two test receivers under varying levels of interference for a SAOCOM-like chirp signal.

be expected to exhibit a similar relative increase in noise during SAR interference. As such, use of an L2C capable receiver would only bring limited added robustness in this specific application compared to existing architectures for semi-codeless L2 tracking.

At a given tracking loop bandwidth, the $\mathrm{C} / \mathrm{N}_{0}$ reduction caused by the interference results in an increase of the measurement noise in inverse proportion to the square-root of the carrier-to-noise-density ratio. Accepting a doubling of the measurement noise, the tolerable drop in $\mathrm{C} / \mathrm{N}_{0}$ during SAR interference amounts to $6 \mathrm{~dB}$. In the given case of adjacent band interference, this threshold is reached for $\mathrm{L} 2$ tracking at interference powers of about $90 \mathrm{~dB}$ relative to the simulated C/A code signal.

\section{IN-BAND INTERFERENCE}

For the assessment of in-band interference, we considered again a chirp signal of $50 \mathrm{MHz}$ bandwidth but with a center frequency of $1225 \mathrm{MHz}$ in the middle of the GPS L2 band. Even though this choice does not exactly match any of the mission profiles described in Table 1, the adopted bandwidth is compatible with the available test equipment and ensures full coverage of the L2 GPS band including the first sidebands. For the pulse duration $(60 \mu \mathrm{s})$ and repeat period $(400 \mu \mathrm{s})$ the same values as in the previous test were employed. Changes in the observed $\mathrm{C} / \mathrm{N}_{0}$ relative to a test without interference are shown in Fig. 7. Since in-band chirp signals are expected to result in stronger degradation of the GPS tracking, the adopted range of interference powers was lowered by $10 \mathrm{~dB}$ compared to the out-of-band test.

Considering again a $6 \mathrm{~dB}$ threshold for the tolerable $\mathrm{C} / \mathrm{N}_{0}$ degradation, both receivers are found to withstand a peak chirp power of $85 \mathrm{~dB}$ at the given duty cycle, which is only slightly less than for the case of adjacent-band interference. Even though the in-band interference would intuitively be considered to be substantially more harmful than the adjacent-band interference, a surprising level of robustness is also obtained in this test. Differences between inband and out-of-band interference are primarily visible at the lower range of simulated chirp signal powers. While the $\mathrm{C} / \mathrm{N}_{0}$ loss of both test receivers stays below $2 \mathrm{~dB}$ for jamming powers of up to $80 \mathrm{~dB}$ in case of adjacent-band interference, the same degradation can already be observed at $55 \mathrm{~dB}$ in case of overlapping center frequencies. 


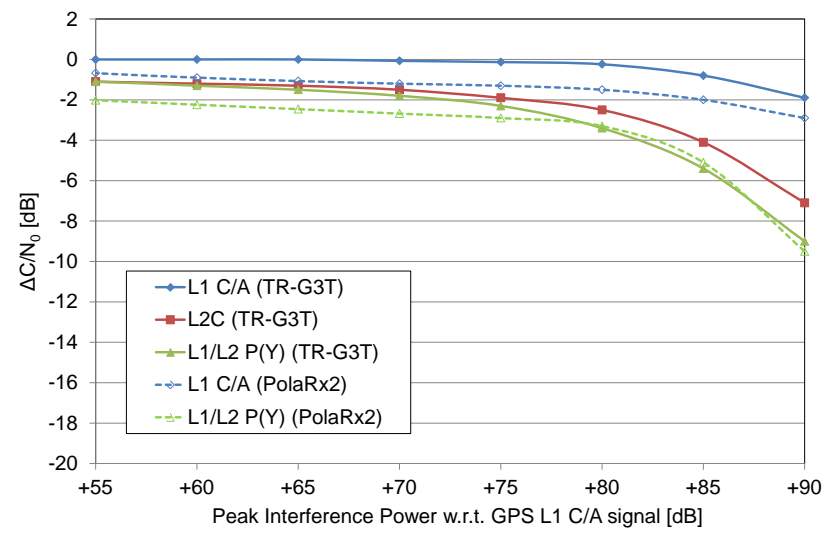

Fig. $7 \mathrm{C} / \mathrm{N}_{0}$ degradation of two test receivers under varying levels of interference for a chirp signal overlaying the GPS L2 frequency.

\section{PULSE BLANKING}

Among others, the effectiveness of pulse blanking for GPS signal tracking depends on the pulse repeat rate and the pulse duty cycle. For the SAR missions described in Table 1 , the pulse duration amounts to $0.02-0.06 \mathrm{~ms}$ and is thus substantially shorter than the code length and coherent integration intervals of the GPS signals. The pulse repeat rates are typically low-integer rational fractions of the code lengths, but due to the Doppler shift of the received signals and frequency offsets of the radar oscillator a strict commensurability of repeat intervals and code lengths is likely to be avoided in practice.

For a first test of GPS tracking under pulse blanking, the $\mathrm{C} / \mathrm{N}_{0}$ and averaged measurement noise of the test receivers was evaluated in a test, where GPS signals were toggled on and off with a pattern matching the chirp duration and period of representative SAR signals, but without injecting any chirp signals into the antenna line. In this way, the response of GPS tracking to periodic interrupts at representative time scales could be assessed independent of the actual interference level.

Table 2 Standard deviation of code $\left(\sigma_{p}\right)$ and carrier phase $\sigma_{\varphi}$ residuals for pulsed blanking with different pulse durations (i.e., off-periods) $T_{\mathrm{p}}$ and pulse repetition interval $T_{\mathrm{r}}$.

\begin{tabular}{lcccccc}
\hline Test case & $\begin{array}{c}T_{\mathrm{p}} \\
{[\mu \mathrm{s}]}\end{array} T_{\mathrm{r}}$ & $\sigma_{p}$ & Ratio \\
{$[\mathrm{m}]$} & {$[\mathrm{dB}]$} & $\begin{array}{c}\sigma_{\varphi} \\
{[\mathrm{mm}]}\end{array}$ & $\begin{array}{c}\text { Ratio } \\
{[\mathrm{dB}]}\end{array}$ \\
\hline No blanking & & & 0.47 & & 3.5 & \\
TanDEM-L & 20 & 200 & 051 & 0.36 & 4.1 & 0.69 \\
SAOCOM & 60 & 400 & 0.54 & 0.60 & 4.3 & 0.90 \\
\hline
\end{tabular}

The change in measurement noise associated with the pulsed GPS signal blanking is summarized in Table 2. All values were obtained with the PolaRx 2 test receiver and re- fer to the ionosphere-free linear combination of L1/L2 P(Y) pseudoranges or L1 C/A and L2 $\mathrm{P}(\mathrm{Y})$ carrier phase observations. The measured increase in noise level amounts to $0.4-0.9 \mathrm{~dB}$ and is in reasonable accord with expectations of $0.45-0.70 \mathrm{~dB}$ for the $10-15 \%$ off-duty cycle as well as measured $\mathrm{C} / \mathrm{N}_{0}$ losses of $1-1.5 \mathrm{~dB}$ for the semi-codeless $\mathrm{P}(\mathrm{Y})$ tracking.

To assess the benefit of pulse blanking in actual interference scenarios, the adjacent-band and in-band interference test scenarios described in the previous sections were used as a baseline. The $\mathrm{R} / \mathrm{F}$ switch in the signal line prior to the low-noise amplifier was toggled synchronously with the simulated SAR signal. Incoming GPS and interference signals were thus blocked in a time window covering the full chirp duration plus a small $1 \mu$ s pre- and post-chirp interval to account for potential transition times. For the remaining part of the SAR repeat period, the switch was opened to allow reception of the unperturbed GPS signals outside the chirp interference.

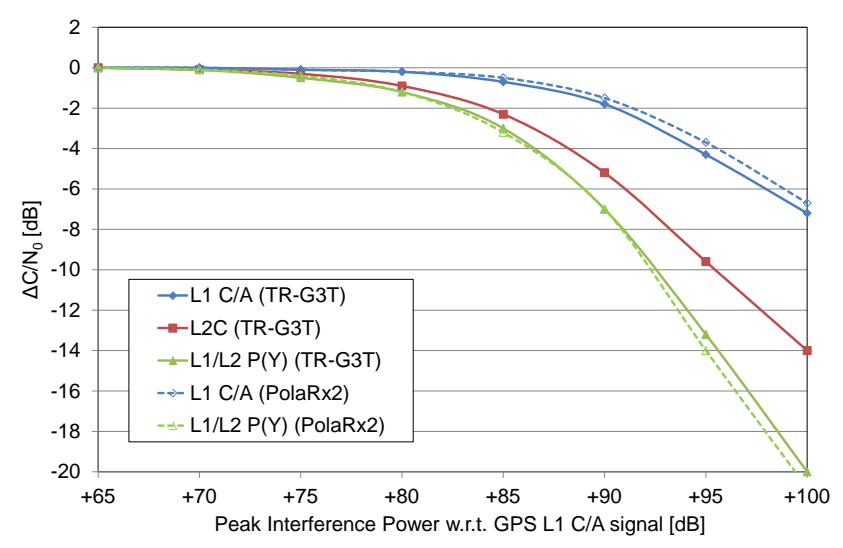

Fig. $8 \mathrm{C} / \mathrm{N}_{0}$ degradation of two test receivers using pulse blanking for a SAOCOM-like chirp signal.

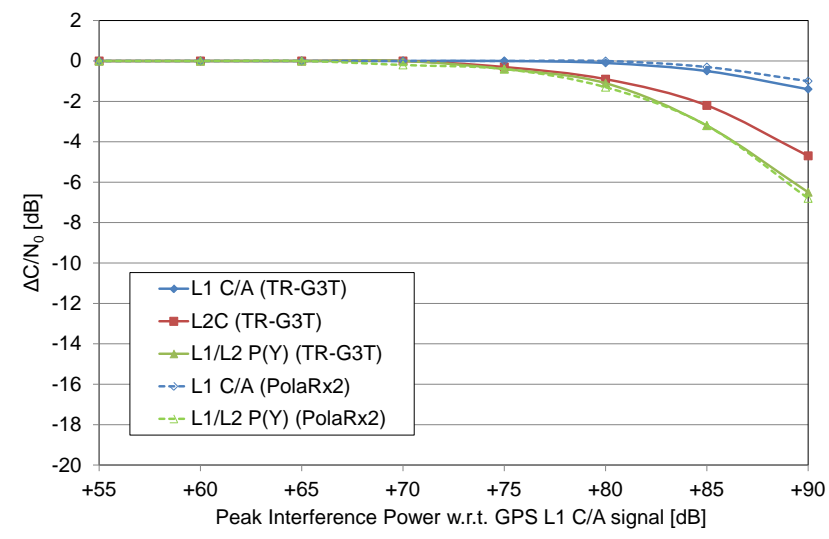

Fig. $9 \mathrm{C} / \mathrm{N}_{0}$ degradation of two test receivers using pulse blanking for a chirp signal overlaying the GPS L2 frequency. 
Results of these tests for interference signals with center frequencies of $1275 \mathrm{MHz}$ and $1225 \mathrm{MHz}$ are shown in Figs. 8 and 9, respectively. Interference power levels are again expressed as peak power of the chirp signal relative to the GPS L1C/A code power and refer to the entrance of the blanking switch. Through pulse blanking, the chirp signal power at the receiver input is reduced by at least $50 \mathrm{~dB}$ with respect to these values. The resulting $\mathrm{C} / \mathrm{N}_{0}$ degradations refer to a reference obtained with $15 \%$ off-duty cycling of the GPS signals to take into account the previously discussed average GPS signal power reduction caused by the pulse blanking itself.

Contrary to expectations, the graphs do not indicate a pronounced reduction of interference effects, but show a strikingly similar jamming sensitivity as in the case without pulse blanking. Compared to Figs. 6 and 7 an almost identical $\mathrm{C} / \mathrm{N}_{0}$ degradation at high jamming powers is obtained, even though the blanking reduces the average chirp power at the receiver input by at least $50 \mathrm{~dB}$. Differences compared to test cases without blanking can only be recognized at interference powers up to $75 \mathrm{~dB}$, where no traces of $\mathrm{C} / \mathrm{N}_{0}$ degradation are discernible when activating the pulse blanking.

These surprising results can best be understood by assuming that the synthesized SAR signal is affected by impurities, which add a low power signal contribution on top of the nominal chirp pulse. The spectral power density of these perturbations appears to be far lower than that of the simulated chirp signal and falls within the noise floor of the available spectrum analyzer. Even though the spectral and temporal characteristics of the impurities remain largely unknown at his stage, the test results suggest that they grow in proportion to the simulated chirp power and cover a notable part of the chirp-free part of the pulse repeat interval. As such, a dedicated pulse blanking device can clearly reduce the impact of the actual chirp pulse, but cannot counteract the adverse impact of additional low power interference signal components outside the pulse period. Furthermore, the very similar GPS signal tracking degradation for in-band and adjacent-band interference observed in our tests provides a strong indication that the impurities are sufficiently broadband to extend well beyond the nominal bandwidth of the actual chirp signal.

\section{SUMMARY AND CONCLUSIONS}

In preparation of future space missions using L-band signals for SAR imaging and interferometry, the impact of SAR interference with GPS tracking was assessed in a GPS signal simulator test bed using synthetic chirp signals. A high level of robustness was found both for the case of adjacent-band interference and in-band interference. For a $50 \mathrm{MHz}$ chirp centered at $1275 \mathrm{MHz}$ (i.e., next to the L2 band), it was found that SAR peak signal powers exceed- ing the received GPS signal level by up to roughly $90 \mathrm{~dB}$ could be handled at a $\mathrm{C} / \mathrm{N}_{0}$ of $6 \mathrm{~dB}$ that corresponds to a doubling of the measurement noise. For a SAR frequency centered on the L2 band, a $85 \mathrm{~dB}$ threshold was obtained that is only moderately lower.

External pulse blanking was found to effectively remove the influence of in-band and adjacent band chirp signals at low and intermediate interference power. However, it proved to be of limited value in case of high-power interference, which can be understood by impurities in the simulated chirp signals. These include relevant interference signal contributions outside the spectral and temporal limits of the pure chirp signal and dominate the observed tracking degradation at simulated chirp power of more than about $80 \mathrm{~dB}$.

Given the fact that current GNSS receivers for space applications have not been specifically designed to support internal pulse-blanking for interference mitigation, the results demonstrate that a substantial robustness against SAR signal interference is already achieved with traditional receiver architectures. Even though technical details of the test receivers have not been disclosed by their manufacturers, it may be suspected that either a fast automatic gain control or a saturation of the analog-to-digital converters during high-power chirp interference helps to implicitly blank this type of interference and enables continued tracking of GPS signals with modest impact on the $\mathrm{C} / \mathrm{N}_{0}$ and the measurement quality. Despite a basic communality of the employed tracking techniques between our test receivers and candidate GPS receivers for future space missions, further tests with actual space receivers are suggested to consolidate the present findings.

The results obtained in the signal simulation testbed may serve as a first reference to decide on safety thresholds (in terms of distance and boresight angle) for mutual SAR illumination of satellites in bistatic SAR formation flying missions. They can also be used to assess the self-interference risk of active SAR satellites for given placements of the SAR and GPS antennas, and to decide on possible needs for band-pass filtering or pulse blanking.

On the other hand, the tests reveal that due attention most be paid to deviations of the actual SAR signal from an idealized chirp. These may cause an additional source of interference and may limit the efficiency of temporal or spectral filtering of the SAR signals even if they contribute only a small fraction of the overall SAR signal power. Since the respective impurities may be difficult to characterize beforehand, dedicated tests using the actual SAR electronics are recommended for a preflight validation of interference mitigation using bandpass filters and/or pulse blanking devices in upcoming L-bad SAR missions. 


\section{REFERENCES}

[1] R. Nakamura, S. Nakamura, N. Kudo, S. Katagiri, "Precise orbit determination for ALOS," in Proc. ISSFD, 24-28 Sep. 2007, Annapolis, MD, 2007.

[2] Y. Arikawa, T. Yamamoto, Y. Kondoh, K. Akiyama, H. Itoh, S. Suzuki, "ALOS-2 orbit control and determination," in Proc. Int. Geoscience and Remote Sensing Symposium (IGARSS), IEEE, pp. 3415-3417, 2014.

[3] A. E. Giraldez, "SAOCOM-1 Argentina L-band SAR mission overview," in Coastal and Marine Applications of SAR Symp, 2003.

[4] L. Frulla, J. Medina, J. Milovich, G. Ortega, M. Thibeault, "SAOCOM Mission Overview," in 2011 CEOS SAR Calibration and Validation Workshop, 2011.

[5] P. Rosen, S. Hensley, S. Shaffer, W. Edelstein, Y. Kim, R. Kumar, T. Misra, R. Bhan, R. Sagi, "The NASA-ISRO SAR (NISAR) mission dual-band radar instrument preliminary design," in 2017 IEEE International Geoscience and Remote Sensing Symposium (IGARSS), IEEE, pp. 38323835, 2017.

[6] N. Gebert, B. C. Dominguez, M. W. J. Davidson, M. D. Martin, P. Silvestrin, "SAOCOM-CS - a passive companion to SAOCOM for single-pass L-band SAR interferometry," in Proc. EUSAR 2014; 10th European Conference on Synthetic Aperture Radar, VDE, pp. 1-4, 2014.

[7] O. Montenbruck, G. Allende-Alba, J. Rosello, M. Tossaint, F. Zangerl, "Precise Orbit and Baseline Determination for the SAOCOM-CS Bistatic Radar Mission," Navigation: Journal of The Institute of Navigation, vol. 65, no. 1, pp. 15-24, 2018.

[8] A. Moreira, G. Krieger, I. Hajnsek, K. Papathanassiou, M. Younis, P. Lopez-Dekker, S. Huber, M. Villano, M. Pardini, M. Eineder, et al., "Tandem-L: A highly innovative bistatic SAR mission for global observation of dynamic processes on the Earth's surface,' IEEE Geoscience and Remote Sensing Magazine, vol. 3, no. 2, pp. 8-23, 2015.

[9] C. Hegarty, A. Van Dierendonck, D. Bobyn, M. Tran, T. Kim, J. Grabowski, "Suppression of pulsed interference through blanking," in Proc. of IAIN World Congress in Association with the US ION 56th Annual Meeting, San Diego, vol. 399, 2000.

[10] A. Simsky, W. De Wilde, T. Willems, D. Mertens, E. Koitsalu, J.-M. Sleewaegen, "First field experience with L5 signals: DME interference reality check," in Proceedings of ION GNSS, pp. 29-37, 2009.

[11] D. Borio, "Swept GNSS jamming mitigation through pulse blanking," in Navigation Conference (ENC), 2016 European, pp. 1-8, 2016.

[12] A. Hein, Processing of SAR data, Springer, 2003.

[13] S. Huber, M. Villano, M. Younis, G. Krieger, A. Moreira, B. Grafmueller, R. Wolters, "Tandem-L: Design concepts for a next-generation spaceborne SAR system,' in EUSAR 2016: 11th European Conference on Synthetic Aperture Radar, Proceedings of, VDE, pp. 1-5, 2016.

[14] A. J. van Dierendonck, "Global Positioning System: Theory and Applications," in Global Positioning System: The- ory and Applications, edited by B. W. Parkinson, P. Enge, P. Axelrad, J. J. Spilker Jr, vol. 1, pp. 329-407, American Institute of Aeronautics and Astronautics, 1996.

[15] O. Montenbruck, M. Garcia-Fernandez, J. Williams, "Performance comparison of semicodeless GPS receivers for LEO satellites," GPS Solutions, vol. 10, no. 4, pp. 249-261, 2006.

[16] J. Wakeman, "Radar Emitter Simulation Using The E8267C PSG Vector Signal Generator," Application Note 58889212EN, Agilent Technologies Inc., 2003.

[17] P. Silvestrin, J. Cooper, "Method of Processing of Signals of a Satellite Positioning System," US Patent 6157 341, 2000.

[18] K. Woo, "Optimum semicodeless carrier-phase tracking of L2," Navigation, vol. 47, no. 2, pp. 82-99, 2000.

[19] A. Hauschild, M. Markgraf, O. Montenbruck, "Flight Results of the NOX Dual-frequency GPS Receiver Payload On-board the TET Satellite," in Proc. ION GNSS+ 2013, ION, pp. 3316-3324, 2013.

[20] A. Helm, M.-P. Hess, M. Minori, A. Gribkov, S. Yudanov, O. Montenbruck, G. Beyerle, L. Cacciapuoti, R. Nasca, "The ACES GNSS Subsystem and its Potential for RadioOccultation and Reflectometry from the International Space Station,", 2009. 